\title{
A Comparison of the Effects of Neuronal Nitric Oxide Synthase and Inducible Nitric Oxide Synthase Inhibition on Cartilage Damage
}

\author{
Nevzat Selim Gokay, ${ }^{1}$ Ibrahim Yilmaz, ${ }^{2}$ Baran Komur, ${ }^{3}$ Ahu Senem Demiroz, \\ Alper Gokce, ${ }^{5}$ Sergülen Dervisoglu, ${ }^{4}$ and Banu Vural Gokay ${ }^{6}$ \\ ${ }^{1}$ Istanbul Esenyurt University, 34510 Istanbul, Turkey \\ ${ }^{2}$ Department of Pharmacovigilance, Materiovigilance and Rational Use of Drugs, Tekirdag State Hospital, Ministry of Health, \\ 59100 Tekirdag, Turkey \\ ${ }^{3}$ Kanuni Sultan Suleyman Training and Research Hospital, Turgut Ozal Street No. 1, Halkali, Kucukcekmece, 34303 Istanbul, Turkey \\ ${ }^{4}$ Istanbul University, Cerrahpasa School of Medicine, Department of Pathology, Istanbul University, 34098 Istanbul, Turkey \\ ${ }^{5}$ Nisantasi University, 34360 Istanbul, Turkey \\ ${ }^{6}$ Department of Anesthesiology and Reanimation, Acıbadem University, 34360 Istanbul, Turkey
}

Correspondence should be addressed to Baran Komur; barankomur@gmail.com

Received 14 March 2016; Revised 3 May 2016; Accepted 16 May 2016

Academic Editor: Patrizia Rovere-Querini

Copyright ( 2016 Nevzat Selim Gokay et al. This is an open access article distributed under the Creative Commons Attribution License, which permits unrestricted use, distribution, and reproduction in any medium, provided the original work is properly cited.

\begin{abstract}
The objective of this study was to investigate the effects of selective inducible nitric oxide synthase and neuronal nitric oxide synthase inhibitors on cartilage regeneration. The study involved 27 Wistar rats that were divided into five groups. On Day 1, both knees of 3 rats were resected and placed in a formalin solution as a control group. The remaining 24 rats were separated into 4 groups, and their right knees were surgically damaged. Depending on the groups, the rats were injected with intra-articular normal saline solution, neuronal nitric oxide synthase inhibitor 7-nitroindazole $(50 \mathrm{mg} / \mathrm{kg})$, inducible nitric oxide synthase inhibitor aminoguanidine $(30 \mathrm{mg} / \mathrm{kg})$, or nitric oxide precursor L-arginine $(200 \mathrm{mg} / \mathrm{kg})$. After 21 days, the right and left knees of the rats were resected and placed in formalin solution. The samples were histopathologically examined by a blinded evaluator and scored on 8 parameters. Although selective neuronal nitric oxide synthase inhibition exhibited significant $(P=0.044)$ positive effects on cartilage regeneration following cartilage damage, it was determined that inducible nitric oxide synthase inhibition had no statistically significant effect on cartilage regeneration. It was observed that the nitric oxide synthase activation triggered advanced arthrosis symptoms, such as osteophyte formation. The fact that selective neuronal nitric oxide synthase inhibitors were observed to have mitigating effects on the severity of the damage may, in the future, influence the development of new agents to be used in the treatment of cartilage disorders.
\end{abstract}

\section{Introduction}

Osteoarthritis (OA) is a progressive disorder that involves cartilage loss. The search continues for a medical or surgical treatment for the cartilage damage that is often blamed for triggering the disorder. In recent years, studies aimed at understanding OA pathophysiology have been conducted. Mechanisms that prevent the formation of the disorder and its advance will also advance treatment methods.
Cartilage loss and subchondral bone resorption are known to develop as a result of a catabolic chemical cascade [1]. Cartilage tissue loss and OA are a result of a breakdown in the balance between cartilage extracellular matrix synthesis and degradation in the catabolic direction [2]. Cytokines that stimulate matrix proteinases (MMP) contribute to the catabolic process [3]. These cytokines also trigger the formation of nitric oxide (NO) in the joints [4]. It has been reported that $\mathrm{NO}$ causes cartilage degradation by increasing 
the effect of IL-1 and triggering apoptosis [5]. NO, which is formed by the oxidation of the guanidino nitrogen of $\mathrm{L}$ arginine, is synthesized by different NO synthases (NOS) in neuronal, endothelial, and inducible manners [6]. It has been shown that inducible NOS (iNOS) is found more often in cells with OA compared to normal cells [6-9]. The inhibition of iNOS decreased the loss of glycosaminoglycan content in an OA model [10]. However, a recent prospective clinical study on OA patients reported that iNOS inhibition had no effect on OA progression [11]. The protective effects of $\mathrm{NO}$ were also emphasized in a recent review about $\mathrm{NO}$ and OA [12]. It has been thought that neuronal NOS (nNOS) might play a more dominant role in the development of the disorder [13]. nNOS activity has been found to be increased in human chondrocytes with OA compared with normal chondrocytes [14]. Few histopathological studies have investigated whether nNOS or iNOS play a more dominant role in the etiopathogenesis of cartilage damage.

From a histopathological perspective, our objective in this study was to investigate and to compare the effectiveness of select nNOS and iNOS inhibitors on cartilage damage in Wistar-type male rats to treat experimentally induced joint damage.

\section{Method}

This study was performed with a live mammal use permit granted by the T.R. Namik Kemal University Experimental Animals Local Ethical Board (Meeting Decision Number 2010/04, dated 01.06.2010), which follows the guidelines of the Turkish Animal Experimentation Regulations. The experimental analyses were repeated 3 times.

2.1. Materials. Wistar-type male rats were obtained from Istanbul University (Experimental Medicine Research Institute, Vakif Gureba Caddesi, 34093 Capa, Istanbul); 7nitroindazole (N7778-5G), amino-guanidine, and L-arginine were obtained from Sigma-Aldrich Chemie GmbH (Steinheim, Germany), and $0.9 \% \mathrm{NaCl}$ (saline solution) was obtained from Biofarma Drug Industry and Commerce, Inc. (Istanbul, Turkey).

2.2. Methods. A total of 27 Wistar-type male rats weighing an average $300 \mathrm{~g}(240-350 \mathrm{~g})$ and with an average age of eighteen weeks were used. All efforts were made to minimize animal suffering and the number of animals used. Experimental Study Design, which is discussed in the National Center for the Replacement, Refinement \& Reduction of Animals in Research (http://www.nc3rs.org.uk/experimental-design), guided us in determining groups and numbers. The animals were granted ad libitum access to food and water and were maintained on a 12-hour light and dark cycle. The rats were randomly separated into 5 groups, with 3 rats in the first group and 6 in each of the other four groups. Rats were kept in different cages in each group at $22.4^{\circ} \mathrm{C}$. All of the procedures were performed in the T.R. Namik Kemal University Experimental Animal Laboratory.

On Day 1 of the experiment, the right and left knees of the 3 rats in the first group were resected, without dissecting

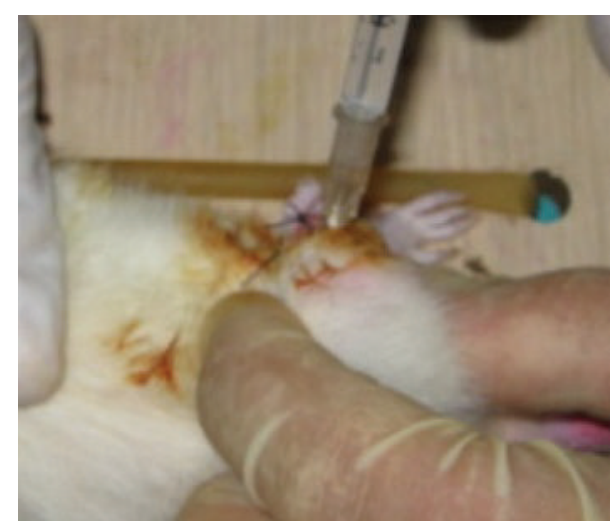

FIGURE 1: Using an insulin injector, active ingredients were injected into the right knees of the rats according to the group.

the muscles or opening the capsule, under anesthesia with administration of an overdose of intraperitoneal sodium pentobarbital. The knees were placed in $10 \%$ formaldehyde and used as the control group (Group $1, n=6)\left(22.4^{\circ} \mathrm{C}\right.$ under normal conditions (UNC)). The rats in Group 1 were sacrificed by cervical dislocation after this procedure. Arthrotomy was performed under anesthesia on the right knees of the remaining 24 rats in 4 groups (Groups 2, 3, 4, and $5 ; n=6$ in each group), and a full-thickness osteochondral defect was created in the medial condyle.

On Day 2 of the experiment, the injections were started, and for 7 days, pharmacological ingredients that were prepared based on the groups were injected twice daily into the defect generated in the right knees of the rats (morning and evening, Table S1 in Supplementary Material available online at http://dx.doi.org/10.1155/2016/7857345). Each time, the same sequence from Group 2 to Group 5 was followed during injections. An insulin injector (100 IU) was used, and the injection was performed into the joint through the medial patellar tendon while the knee was at $90^{\circ}$ flexion (Figure 1). The needle's entry into the joint space was checked by the loss of resistance to the needle.

On Day 29 of the experiment, the defective right knees and the intact left knees of all rats (to determine the systemic effects of the injected active ingredients) were resected without dissecting the muscles or opening the capsules, under anesthesia with administration of an overdose of intraperitoneal sodium pentobarbital. All of the samples were preserved in $10 \%$ formaldehyde $\left(22.4^{\circ} \mathrm{C} \mathrm{UNC}\right)$. Then, the rats were sacrificed by cervical dislocation.

The obtained materials were coded and sent to the Pathology Laboratory of Istanbul University's Cerrahpaşa Medical School for histopathological evaluation.

2.3. Applied Surgical Technique. All surgical procedures were performed under aseptic conditions by a single surgeon (NSG). The rats were anesthetized with an intraperitoneal administration of $30 \mathrm{mg} / \mathrm{kg}$ sodium pentobarbital, and their right knees were shaved. Entry to the rats' right knees was through an anterior skin incision. The capsule was opened using a medial parapatellar approach, and care was taken to 

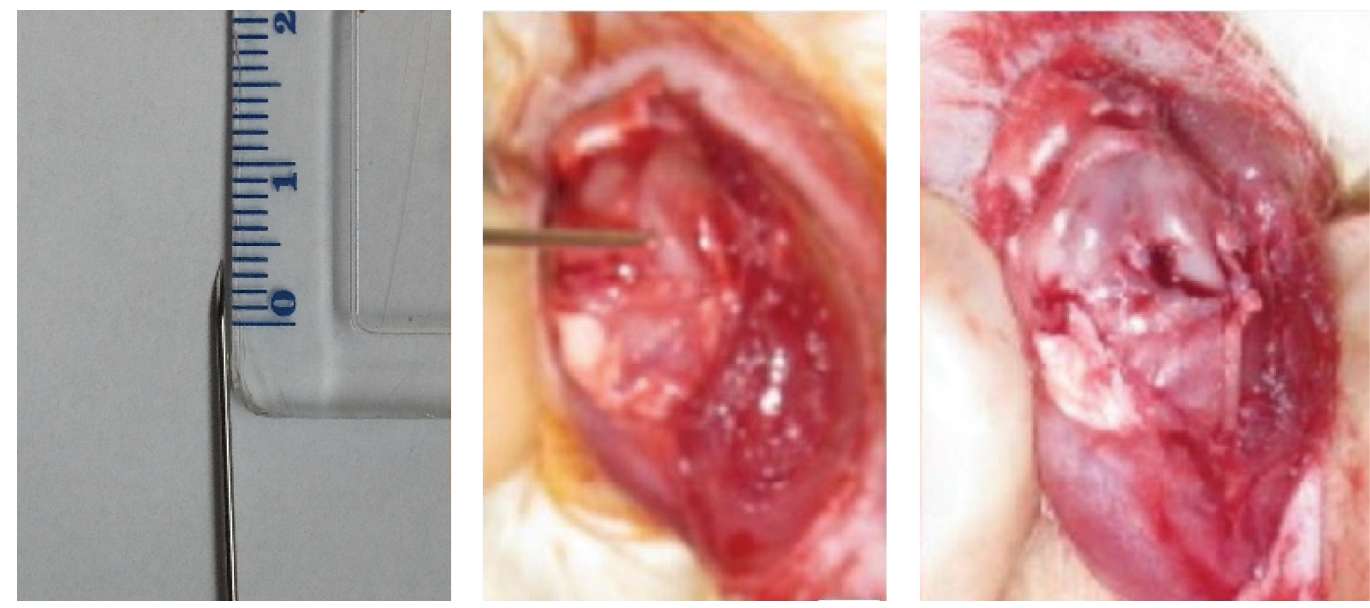

FIGURE 2: Full-thickness cartilage defects were generated with a 21-gauge needle.

prevent iatrogenic joint injury. A full-thickness osteochondral defect $(0.8 \mathrm{~mm}$ thickness $\times 4 \mathrm{~mm}$ depth $)$ was generated in the medial condyle with the tip of an injection needle (21gauge sterile needle, Beybi Plastik Fab. San. AŞ., Istanbul, Turkey) [15-17]. Lesions were created with a 21-gauge needle ( $0.8 \mathrm{~mm}$ in diameter, $4 \mathrm{~mm}$ bevel length) to the lateral wall of the medial condyle at the level of the intersection of the trochlear groove and the intercondylar notch (Figure 2). The needle was inserted in one attempt, until the bevel of the needle was entirely inside the femur. The skin was closed and the procedure ended.

2.4. Histopathological Evaluation. After routine tissue processing, the tissue samples were embedded in paraffin blocks. Sections of $5 \mu \mathrm{m}$ thickness were stained with hematoxylineosin and examined with a light microscope. The pathologist who performed the examinations was blinded to the experimental groups, drugs used, and which knee joint was being examined. The histological sections were evaluated and scored based on the condition of the surface layer, clone formation of the chondrocytes, single cell death in the chondrocytes, decreases in cartilage layer thickness, increases in subchondral bone thickness, chronic synovitis findings, and synovial cyst and osteophyte formations (Table S2) [18].

2.5. Statistical Evaluation. SPSS 22.0 (IBM statistics for Windows version 22, IBM Corporation, Armonk, New York, United States) was used to analyze the data. The MannWhitney $U$ Test was used to compare two independent samples. The Kruskal-Wallis $H$ Test was used to compare between multiple groups, and the nonparametric Post hoc Test (Miller 1966) was used for post hoc analyses. Pearson chi-square, linear-by-linear association, and Fisher exact tests were tested using the Monte Carlo simulation technique to compare categorical data. The Monte Carlo simulation technique results were used in all analyses. Quantitative data are given in tables as the mean $\pm \mathrm{SD}$ (standard deviation), median \pm IQR (interquartile range), and median and range (maximum-minimum). Categorical data are given as $n$ (numbers) and percentages (\%). The data were analyzed in $95 \%$ confidence intervals, and statistical significance was set at $P<0.05$.

\section{Results}

No complications were encountered during the surgeries, injections, and follow-up period. The postoperative behavior of all rats was no different than that observed preoperatively, with normal feeding and behavior towards handlers.

As mentioned in methods section, both knees were resected without any defects on Day 1 of the experiment in Group 1, intra-articular SF (15 mg/kg/day) was applied to the defect-induced right knees for 7 days in Group 2, intraarticular SF (15 mg/kg/day) + 7-nitroindazole (50 mg/kg/day) was applied to the defect-induced right knees for 7 days in Group 3, intra-articular SF (15 mg/kg/day) + aminoguanidine $(30 \mathrm{mg} / \mathrm{kg} /$ day $)$ was applied to the defect-induced right knees for 7 days in Group 4, and intra-articular SF $(15 \mathrm{mg} / \mathrm{kg} /$ day $)+\mathrm{L}$-arginine $(200 \mathrm{mg} / \mathrm{kg} /$ day $)$ was applied to the defect-induced right knees for 7 days in Group 5 (Table S1).

Comparing the right and left knees of Group 2 to Group 5, there was statistically significantly worse results in right knees of Group 5, according to all parameters (Table S3). There were no statistically significant differences between the right and left knees of Group 3 and Group 4 according to chronic synovitis parameters $(P=0.316$ and $P=0.235$, resp.). According to surface characteristics, there were statistically significantly worse results in the right knees of each group $(P=0.002)$.

There were significantly different results observed for each parameter when Group 1 knees and the right knees of all other groups were compared (Table S4). While there was no clone formation in Group 1, there were 2 small clones and 4 large clones in Group 5. Large clones were mostly observed in Group 5 compared with 2 in Group 2, 0 in Group 3, and 3 in Group 4. Osteophyte formation was also only observed in Group $5(P=0.003)$ (Figures 3-6). 


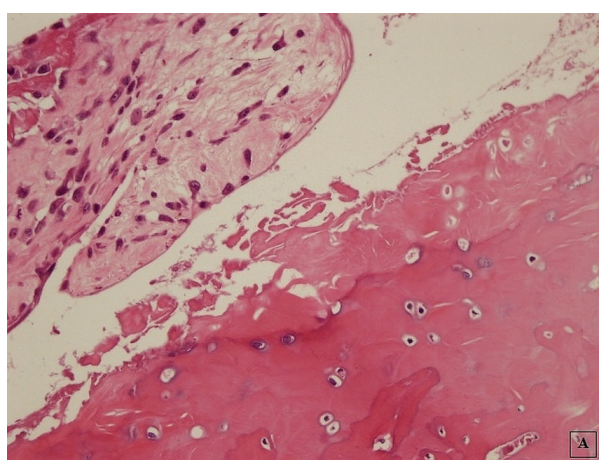

(a)

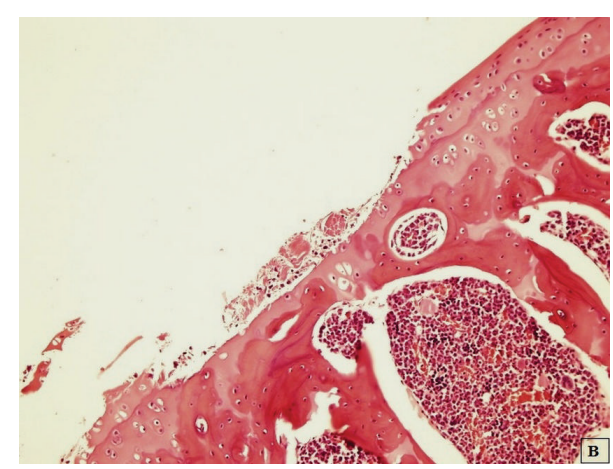

(b)

Figure 3: Predominant fissuration ((a) a histological section taken from the right knee of a rat in Group 3, HE $\times 400)$ and erosion ((b) a histological section taken from the right knee of a rat in Group 3, HE $\times 100)$ were observed in the right (defective) knees of the rats in Group 3.

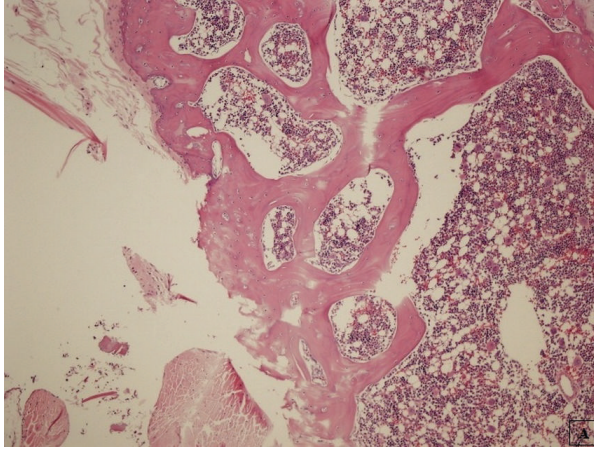

(a)

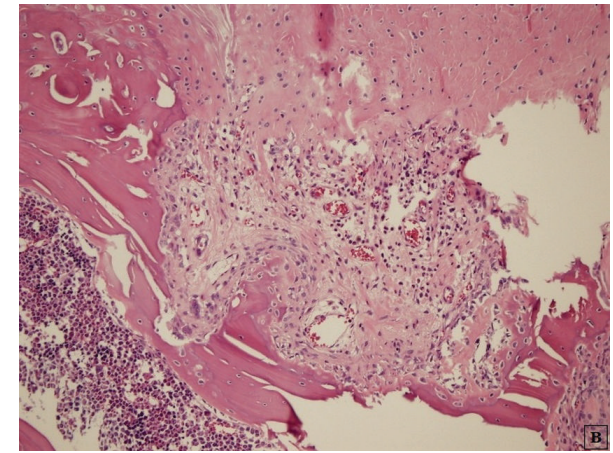

(b)

FIGURE 4: Predominant denudation ((a) a histological section from the right knee of a rat from Group 4, HE $\times 100)$ and deformation ((b) a histological section from the right knee of a rat from Group 5, HE $\times 200$ ) were observed in the right (defective) knees of the rats from Groups 4 and 5 .

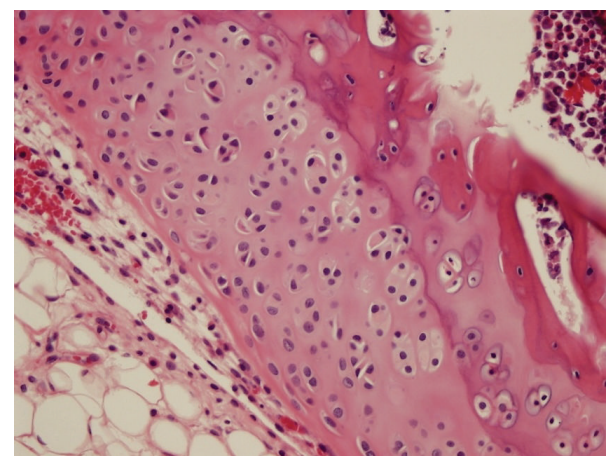

Figure 5: A histological section taken from the right knee of a rat from Group 4 shows large clones consisting of large numbers of chondrocytes $(\mathrm{HE} \times 400)$.

In Groups 2, 3, 4, and 5, statistically significant worse surface characteristic results were observed compared to those in Group $1(P<0.001)$. Statistically significant worse results were found compared to those in Group $3(P<0.001)$ in Groups 4 and 5 (Table S4, Figure 7).

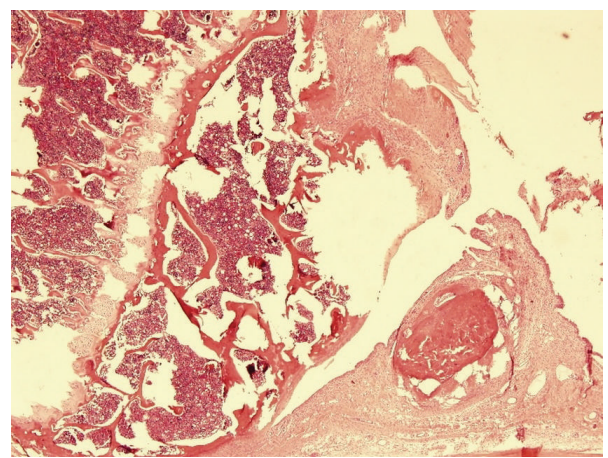

FIGURE 6: A histological section taken from the right knee of a rat from Group 5 shows the development of an osteophyte on the right bottom $(\mathrm{HE} \times 100)$.

\section{Discussion}

In this study, we formed a mechanical OA model in Wistar rats and then attempted to cure the pathology with different agents. The histopathologic evaluations were blindly performed by a pathologist who is an expert in musculoskeletal 


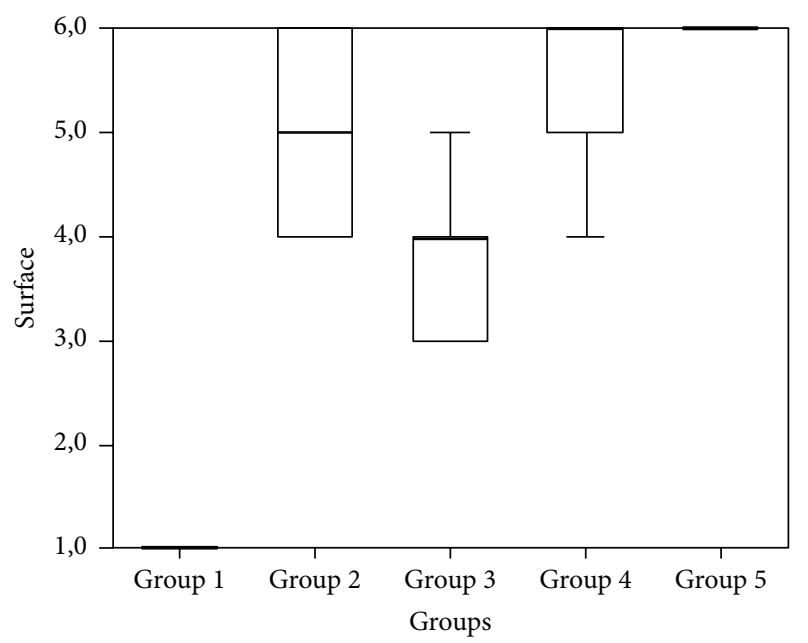

FIGURE 7: A surface characteristics distribution graph for Group 1 and the right (defective) knees of all other groups (Groups 2-5).

pathology. There are a number of limitations in this study. The chosen species may be a bit too small to indicate that the results are also appropriate for humans. We chose this species because of their advantages in terms of cost and housing. Additionally, there are rat OA models used to study the treatment of OA in the literature [19]. The surgical technique we used to form an OA model was recently published [17]. The placement of defect lesions was guided by human approximation. Needle punching was performed in a single attempt, and care was taken to improve standardization. The homogeneous results observed within the groups helped us to overcome this bias. The histopathologic evaluation and scoring of the severity of cartilage damage were performed by the same observer with an individual and previously published scoring system [18]. The system has some alterations other than Osteoarthritis Research Society International (OARSi) recommendations but evaluates the same topics, surface characteristics, osteophyte formation, synovial changes, and cartilage and bone thickness [20]. The scoring system used in this study depends on yes or no answers; therefore, this system seems more objective for standardization to us. Furthermore, another rationale for us to use this scoring system is that it is the system with which our observer is experienced.

$\mathrm{OA}$ is a disorder that involves progressive deterioration and cartilage loss and is characterized by the final disruption of joint functions. This deterioration of the cartilage is triggered by the synthesis of some catabolic substances in the diarthrodial joints. MMP and NO, which are thought to play important roles in the catabolic process, are released through the stimulation of cytokines, such as IL- 1 and TNF- $\alpha$, and NOS [21].

$\mathrm{NO}$ is synthesized from L-arginine amino acids by 2 different NOS that are referred to by the name of the cells in which they exist. In vascular cells, NOS is called endothelial NOS (eNOS), and in nerve cells, it is called nNOS. In addition to their systemic effects, it has been reported that both NOS play roles in the proliferation of the cartilage tissue during its development $[22,23]$. In addition, the existence of a third NOS, iNOS, which is not normally present in cells but is induced as a result of the secreted cytokines, has been discovered $[24,25]$. Similar to the others, iNOS uses the primary substance of L-arginine amino acid in NO synthesis and is thought to be responsible for the tissue breakdown in various disorders, including cartilage breakdown in OA.

In inflammatory disorders, many proinflammatory cytokines and iNOS are secreted from the synovial membrane, but in the case of OA, they are expressed by the affected cartilage cells more than the synovial membrane [26]. Furthermore, it has been shown that the isolated cartilage cultures obtained from OA patients synthesized greater amounts of NO compared with cartilage cultures obtained from patients with rheumatoid arthritis [27]. This condition has been attributed to an excess production of cytokines in cartilage cells with $\mathrm{OA}$ and the upregulation of NOS [4, 6, 27-30]. Mechanical stress has also been shown to increase the production of NO from chondrocytes [31]. More NO is secreted from arthritic cartilage tissue compared to normal cartilage tissue $[6,32]$.

Although the literature has demonstrated that iNOS and $\mathrm{NO}$ are found in increased amounts in cartilage cells with $\mathrm{OA}$, there is not enough information on the role $\mathrm{NO}$ plays in the development of the disorder. The fact that NO suppresses the synthesis of the IL-1 receptor antagonist and increases the effectiveness of IL-1 results in it being blamed for the pathogenesis [33]. Furthermore, it is believed that NO's activation of matrix metalloproteinases also plays a role in the pathogenesis [34]. In addition, it is also believed that, in an indirect manner, NO contributes to the catabolic process by increasing the expression of cytokines, such as IL-18 and IL1 [35]. NO has also been reported to trigger cell death [36]. Interestingly, our study observed cell death in the samples in which we had inhibited NO with iNOS and nNOS and also in those in which we increased NO, which was significantly greater compared with that in the right knees of the rats in Group $2(P<0.001)$.

In some studies that attempted to suppress the iNOS gene, it was concluded that iNOS does not play an indicative role in the course of OA; in fact, it exhibits a prophylactic effect on the cartilage by increasing the synthesis of proteoglycan and decreasing breakdown $[37,38]$. Tamura et al. stated that basic fibroblast growth factor is secreted into the environment through the influence of $\mathrm{NO}$, and as a result, $\mathrm{NO}$ has indirect favorable effects on cell proliferation and angiogenesis [39]. These studies indicate that NO might be a substance synthesized by activating the iNOS pathway after damage to the joint to protect the cartilage and to increase its regeneration. Häuselmann et al. determined that different cartilage layers synthesize different amounts of NO in response to IL-1 stimulation and that different layers react differently to NOS inhibition [40]. The authors showed that $\mathrm{N}^{\mathrm{G}}$-monomethyl-Larginine (L-NMA) completely nullifies the inhibitory effect of IL-1 on proteoglycan in the deeper layers of the cartilage. They also determined that the stimulatory effect of IL-1 on NO decreases with age. The authors believe that, based on these results, NO might play a prophylactic role in the proteoglycan catabolism. Bezerra et al. disclosed that, in an OA model 
created with zymosan, selective iNOS inhibitors or nonselective NOS inhibitors reduced inflammation but did not affect the glycosaminoglycan loss [41]. In support of these findings, it was also observed in our study that there was no statistically significant difference in surface characteristics in the samples in which iNOS was blocked and NO was induced $(P<0.001)$.

These findings show that the role of NO in the joint is more complicated than previously assumed, and more studies are needed to fully understand its efficacy. The majority of studies on NO and OA have been based on the selective inhibition of iNOS, the secretion of which has been found to increase in cartilage with OA. Because the protective effect of iNOS on joint cartilage has been determined in some studies, the question remains whether the other NOSs may have a predominant effect on OA etiopathogenesis. In a study investigating the effects of nNOS inhibition on endochondral bone development, a decrease in the proliferation of chondrocytes upon the blockage of the nNOS gene was reported [23]. Pozza et al. investigated the role of the peripheral nervous system in $\mathrm{OA}$ and disclosed that neuronal $\mathrm{NO}$ affects the development of the disorder [13].

There are some studies which evaluate NO role in immunologic pathways. Paolucci et al. investigated NO inhibition in TNF-alpha regulation in endocytosis of human dendritic cell [42]. They found antagonist effect with TNFalpha and NO. In another study of Falcone et al., they investigated protective effect of $\mathrm{NO}$ in apoptosis of dendritic cells [43]. These kinds of studies also refer to protective effect of $\mathrm{NO}$ in cellular mechanism.

Our study demonstrated that the suppression of NO synthesis by iNOS in rats with an experimental cartilage defect did not affect cartilage regeneration. However, a distinct improvement was observed in the surface characteristics in the knees of the rats in which the synthesis of NO was suppressed with nNOS. Deterioration in all pathological parameters was observed in the group in which NO synthesis was induced, and unlike other groups, osteophytes developed in the knees of the rats in this group.

\section{Conclusion}

These findings show that different NOSs affect cartilage metabolism differently. In our study, it was histopathologically shown that, similar to the results obtained by Pozza et al. [13], nNOS inhibition has positive effects on cartilage healing, whereas iNOS inhibition has no significant effect. These findings may provide guidance for the development of new methods to treat $\mathrm{OA}$ in the future. New studies are needed to investigate the influence of different NOSs in NO synthesis and OA etiopathogenesis.

\section{Disclosure}

The authors alone are responsible for the content and writing of the paper.

\section{Competing Interests}

The authors report no conflict of interests.

\section{Authors' Contributions}

Nevzat Selim Gokay was responsible for experiment design, surgical interventions, performing cartilage damage, intraarticular injections, and editing of the paper. Ibrahim Yilmaz carried out experiment design, drug management (preparation), obtaining and transporting the samples, and drafting the paper. Ahu Senem Demiroz was responsible for histopathological evaluation, statistical study, and revising the paper. Baran Komur was responsible for interpretation of the data and drafting, revising, and English editing of the paper. Alper Gokce was responsible for experiment design, performing cartilage damage, and control of the study stages. Sergülen Dervisoglu was responsible for interpretation of the data, histopathological evaluation, and review of the paper. Banu Vural Gokay was responsible for study design, anesthesia interventions, postoperative care of the animals, and English editing of the paper. All authors have read and approved the final submitted paper.

\section{Acknowledgments}

The authors thank the Turkish Orthopedic Society (TOTBID) for their grants (28.02.2011 date, no. 11) in support of this study. They also thank Oguz Yacan and http://www.medicalstatistic.com/ for help with the statistical analysis.

\section{References}

[1] D. Jang and G. A. C. Murrell, "Nitric oxide in arthritis," Free Radical Biology and Medicine, vol. 24, no. 9, pp. 1511-1519, 1998.

[2] K. D. Brandt, C. W. Slemenda, and A. Osteoarthritis, "Epidemiology, pathology and pathophysiology," in Primer on the Rheumatic Diseases, H. R. Schumacher, J. H. Klippel, and W. J. Koopman, Eds., Arthritis Foundation, Atlanta, Ga, USA, 1993.

[3] C. I. Westacott and M. Sharif, "Cytokines in osteoarthritis: mediators or markers of joint destruction?" Seminars in Arthritis and Rheumatism, vol. 25, no. 4, pp. 254-272, 1996.

[4] K. Vuolteenaho, T. Moilanen, N. Al-Saffar, R. G. Knowles, and E. Moilanen, "Regulation of the nitric oxide production resulting from the glucocorticoid-insensitive expression of iNOS in human osteoarthritic cartilage," Osteoarthritis and Cartilage, vol. 9, no. 7, pp. 597-605, 2001.

[5] M. L. Tiku, S. Gupta, and D. R. Deshmukh, "Aggrecan degradation in chondrocytes is mediated by reactive oxygen species and protected by antioxidants," Free Radical Research, vol. 30, no. 5, pp. 395-405, 1999.

[6] R. M. Clancy, A. R. Amin, and S. B. Abramson, "The role of nitric oxide in inflammation and immunity," Arthritis and Rheumatism, vol. 41, no. 7, pp. 1141-1151, 1998.

[7] E. W. St Clair, "Nitric oxide-friend or foe in arthritis?" Journal of Rheumatology, vol. 25, no. 8, pp. 1451-1453, 1998.

[8] A. Karan, M. A. Karan, P. Vural et al., "Synovial fluid nitric oxide levels in patients with knee osteoarthritis," Clinical Rheumatology, vol. 22, no. 6, pp. 397-399, 2003.

[9] D. Casagrande, J. P. Stains, and A. M. Murthi, "Identification of shoulder osteoarthritis biomarkers: comparison between shoulders with and without osteoarthritis," Journal of Shoulder and Elbow Surgery, vol. 24, no. 3, pp. 382-390, 2015. 
[10] V. Balaganur, N. N. Pathak, M. C. Lingaraju et al., "Effect of Smethylisothiourea, an inducible nitric oxide synthase inhibitor, in joint pain and pathology in surgically induced model of osteoarthritis," Connective Tissue Research, vol. 55, no. 5-6, pp. 367-377, 2014.

[11] M.-P. H. Le Graverand, R. S. Clemmer, P. Redifer et al., "A 2year randomised, double-blind, placebo-controlled, multicentre study of oral selective iNOS inhibitor, cindunistat (SD-6010), in patients with symptomatic osteoarthritis of the knee," Annals of the Rheumatic Diseases, vol. 72, no. 2, pp. 187-195, 2013.

[12] S. B. Abramson, "Osteoarthritis and nitric oxide," Osteoarthritis and Cartilage, vol. 16, no. 2, pp. S15-S20, 2008.

[13] M. Pozza, C. Bettelli, F. Magnani, M. T. Mascia, E. Manzini, and L. Calzà, "Is neuronal nitric oxide involved in adjuvant-induced joint inflammation?" European Journal of Pharmacology, vol. 359, no. 1, pp. 87-93, 1998.

[14] L. Ramage, M.-A. Martel, G. E. Hardingham, and D. M. Salter, "NMDA receptor expression and activity in osteoarthritic human articular chondrocytes," Osteoarthritis and Cartilage, vol. 16, no. 12, pp. 1576-1584, 2008.

[15] K. Kettunen and P. Rokkanen, "The repair of a full thickness articular defect. An experimental study on growing rats," Annales Chirurgiae et Gynaecologiae, vol. 62, no. 3, pp. 166-168, 1973.

[16] S. Mendelson, P. Wooley, D. Lucas, and D. Markel, "The effect of hyaluronic acid on a rabbit model of full-thickness cartilage repair," Clinical Orthopaedics and Related Research, no. 424, pp. 266-271, 2004.

[17] D. M. Knapik, R. K. Harrison, R. A. Siston, S. Agarwal, and D. C. Flanigan, "Impact of lesion location on the progression of osteoarthritis in a rat knee model," Journal of Orthopaedic Research, vol. 33, no. 2, pp. 237-245, 2015.

[18] B. E. Gencosmanoglu, M. Eryavuz, and S. Dervisoglu, "Effects of some nonsteroidal anti-inflammatory drugs on articular cartilage of rats in an experimental model of osteoarthritis," Research in Experimental Medicine, vol. 200, no. 3, pp. 215-226, 2001.

[19] R. R. Castro, F. Q. Cunha, F. S. Silva Jr., and F. A. C. Rocha, "A quantitative approach to measure joint pain in experimental osteoarthritis-evidence of a role for nitric oxide," Osteoarthritis and Cartilage, vol. 14, no. 8, pp. 769-776, 2006.

[20] N. Gerwin, A. M. Bendele, S. Glasson, and C. S. Carlson, "The OARSI histopathology initiative-recommendations for histological assessments of osteoarthritis in the rat," Osteoarthritis and Cartilage, vol. 18, supplement 3, pp. S24-S34, 2010.

[21] J. Martel-Pelletier, "Pathophysiology of osteoarthritis," Osteoarthritis and Cartilage, vol. 6, no. 6, pp. 374-376, 1998.

[22] Q. Yan, Q. Feng, and F. Beier, "Endothelial nitric oxide synthase deficiency in mice results in reduced chondrocyte proliferation and endochondral bone growth," Arthritis \& Rheumatism, vol. 62, no. 7, pp. 2013-2022, 2010.

[23] Q. Yan, Q. Feng, and F. Beier, "Reduced chondrocyte proliferation, earlier cell cycle exit and increased apoptosis in neuronal nitric oxide synthase-deficient mice," Osteoarthritis and Cartilage, vol. 20, no. 2, pp. 144-151, 2012.

[24] A. K. Nussler, M. Di Silvio, T. R. Billiar et al., "Stimulation of the nitric oxide synthase pathway in human hepatocytes by cytokines and endotoxin," Journal of Experimental Medicine, vol. 176, no. 1, pp. 261-264, 1992.

[25] A. R. Amin, M. Attur, and S. B. Abramson, "Nitric oxide synthase and cyclooxygenases: distribution, regulation, and intervention in arthritis," Current Opinion in Rheumatology, vol. 11, no. 3, pp. 202-209, 1999.

[26] C. Melchiorri, R. Meliconi, L. Frizziero et al., "Enhanced and coordinated in vivo expression of inflammatory cytokines and nitric oxide synthase by chondrocytes from patients with osteoarthritis," Arthritis and Rheumatism, vol. 41, no. 12, pp. 2165-2174, 1998.

[27] I. Mazzetti, B. Grigolo, L. Pulsatelli et al., "Differential roles of nitric oxide and oxygen radicals in chondrocytes affected by osteoarthritis and rheumatoid arthritis," Clinical Science, vol. 101, no. 6, pp. 593-599, 2001.

[28] A. R. Amin, P. E. Di Cesare, P. Vyas et al., "The expression and regulation of nitric oxide synthase in human osteoarthritisaffected chondrocytes: evidence for up-regulated neuronal nitric oxide synthase," Journal of Experimental Medicine, vol. 182, no. 6, pp. 2097-2102, 1995.

[29] H. Sakurai, H. Kohsaka, M.-F. Liu et al., "Nitric oxide production and inducible nitric oxide synthase expression in inflammatory arthritides," The Journal of Clinical Investigation, vol. 96, no. 5, pp. 2357-2363, 1995.

[30] J. Stadler, M. Stefanovic-Racic, T. R. Billiar et al., "Articular chondrocytes synthesize nitric oxide in response to cytokines and lipopolysaccharide," Journal of Immunology, vol. 147, no. 11, pp. 3915-3920, 1991.

[31] B. Fermor, J. B. Weinberg, D. S. Pisetsky, M. A. Misukonis, A. J. Banes, and F. Guilak, "The effects of static and intermittent compression on nitric oxide production in articular cartilage explants," Journal of Orthopaedic Research, vol. 19, no. 4, pp. 729-737, 2001.

[32] J.-P. Pelletier, F. Mineau, P. Ranger, G. Tardif, and J. MartelPelletier, "The increased synthesis of inducible nitric oxide inhibits IL-1ra synthesis by human articular chondrocytes: possible role in osteoarthritic cartilage degradation," Osteoarthritis and Cartilage, vol. 4, no. 1, pp. 77-84, 1996.

[33] J. P. Pelletier, F. Mineau, P. Ranger, and J. Martel-Pelletier, “The synthesis of nitric oxide induced by IL-1 in human chondrocytes markedly reduced the synthesis of IL-1 receptor antagonist (IL$1 \mathrm{ra}$ ): a possible role in osteoarthritic cartilage degradation," Transactions of the Orthopaedic Research Society, vol. 20, article 352, 1995.

[34] G. A. C. Murrell, D. Jang, and R. J. Williams, "Nitric oxide activates metalloprotease enzymes in articular cartilage," Biochemical and Biophysical Research Communications, vol. 206, no. 1, pp. 15-21, 1995.

[35] C. Boileau, J. Martel-Pelletier, F. Moldovan et al., "The in situ upregulation of chondrocyte interleukin-1-converting enzyme and interleukin-18 levels in experimental osteoarthritis is mediated by nitric oxide," Arthritis and Rheumatism, vol. 46, no. 10, pp. 2637-2647, 2002.

[36] S. Hashimoto, K. Takahashi, D. Amiel, R. D. Coutts, and M. Lotz, "Chondrocyte apoptosis and nitric oxide production during experimentally induced osteoarthritis," Arthritis and Rheumatism, vol. 41, no. 7, pp. 1266-1274, 1998.

[37] D. M. Visco, D. S. Fletcher, C. J. Orevillo et al., "NOS 2 deficient mice are susceptible to collagen-induced arthritis," NOS, vol. 43, p. 416, 1997.

[38] M. Stefanovic-Racic, T. I. Morales, D. Taskiran, L. A. McIntyre, and C. H. Evans, "The role of nitric oxide in proteoglycan turnover by bovine articular cartilage organ cultures," Journal of Immunology, vol. 156, no. 3, pp. 1213-1220, 1996.

[39] T. Tamura, T. Nakanishi, Y. Kimura et al., "Nitric oxide mediates interleukin-1-induced matrix degradation and basic fibroblast 
growth factor release in cultured rabbit articular chondrocytes: a possible mechanism of pathological neovascularization in arthritis," Endocrinology, vol. 137, no. 9, pp. 3729-3737, 1996.

[40] H. J. Häuselmann, M. Stefanovic-Racic, B. A. Michel, and C. H. Evans, "Differences in nitric oxide production by superficial and deep human articular chondrocytes: implications for proteoglycan turnover in inflammatory joint diseases," Journal of Immunology, vol. 160, no. 3, pp. 1444-1448, 1998.

[41] M. M. Bezerra, S. D. Brain, S. Greenacre et al., "Reactive nitrogen species scavenging, rather than nitric oxide inhibition, protects from articular cartilage damage in rat zymosaninduced arthritis," British Journal of Pharmacology, vol. 141, no. 1, pp. 172-182, 2004.

[42] C. Paolucci, P. Rovere, C. De Nadai, A. A. Manfredi, and E. Clementi, "Nitric oxide inhibits the tumor necrosis factor $\alpha$ regulated endocytosis of human dendritic cells in a cyclic GMPdependent way," Journal of Biological Chemistry, vol. 275, no. 26, pp. 19638-19644, 2000.

[43] S. Falcone, C. Perrotta, C. D. Palma et al., "Activation of acid sphingomyelinase and its inhibition by the nitric oxide/cyclic guanosine $3^{\prime}, 5^{\prime}$-monophosphate pathway: key events in Escherichia coli-elicited apoptosis of dendritic cells," The Journal of Immunology, vol. 173, no. 7, pp. 4452-4463, 2004. 


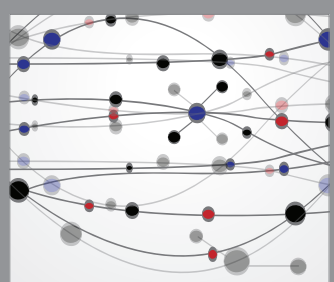

The Scientific World Journal
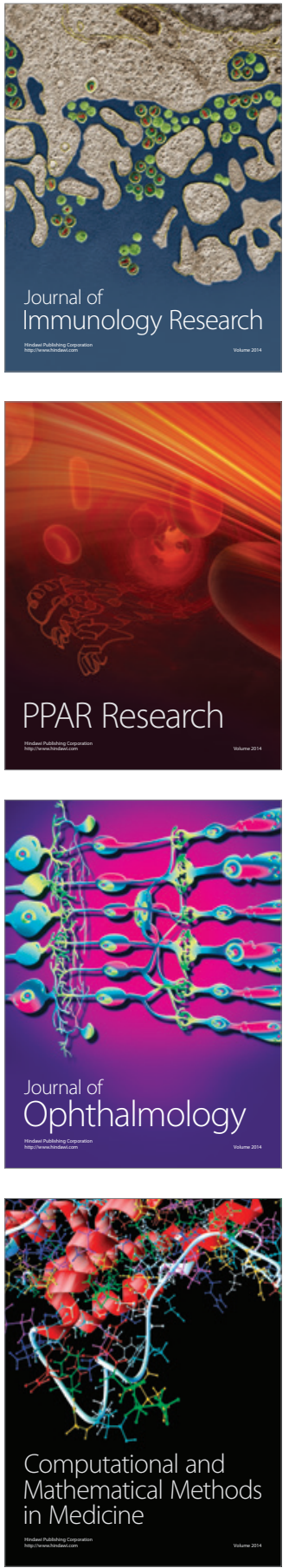

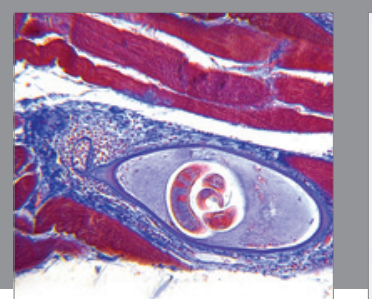

Gastroenterology Research and Practice

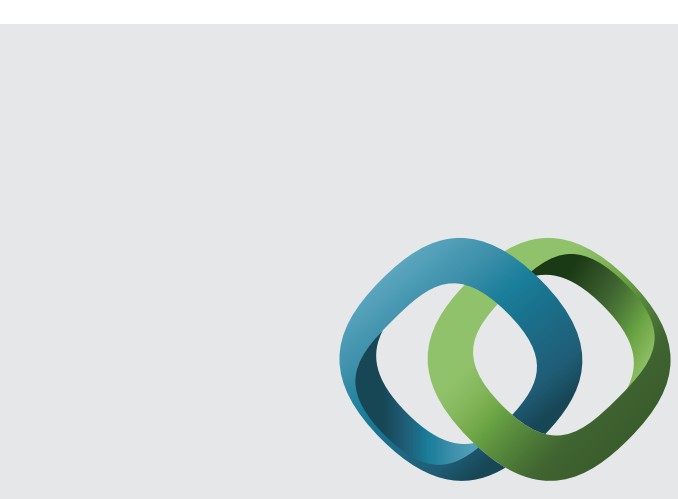

\section{Hindawi}

Submit your manuscripts at

http://www.hindawi.com
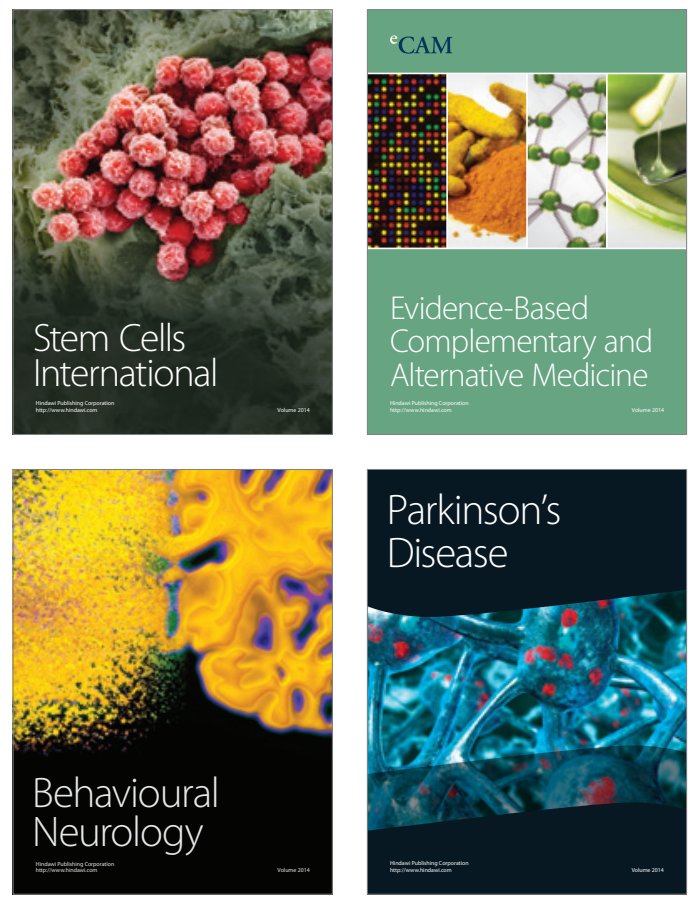
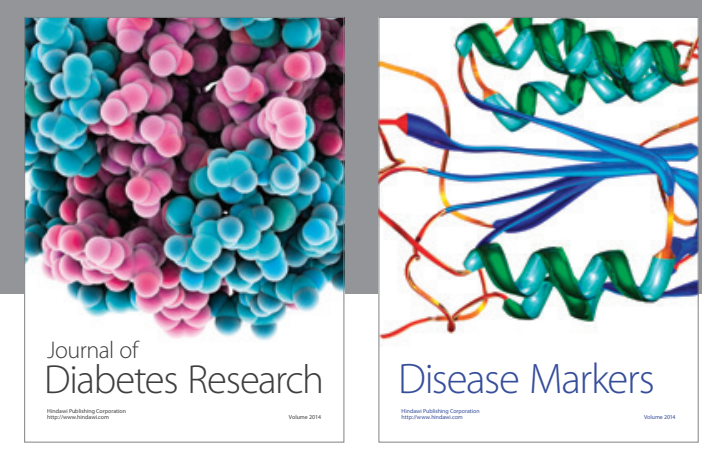

Disease Markers
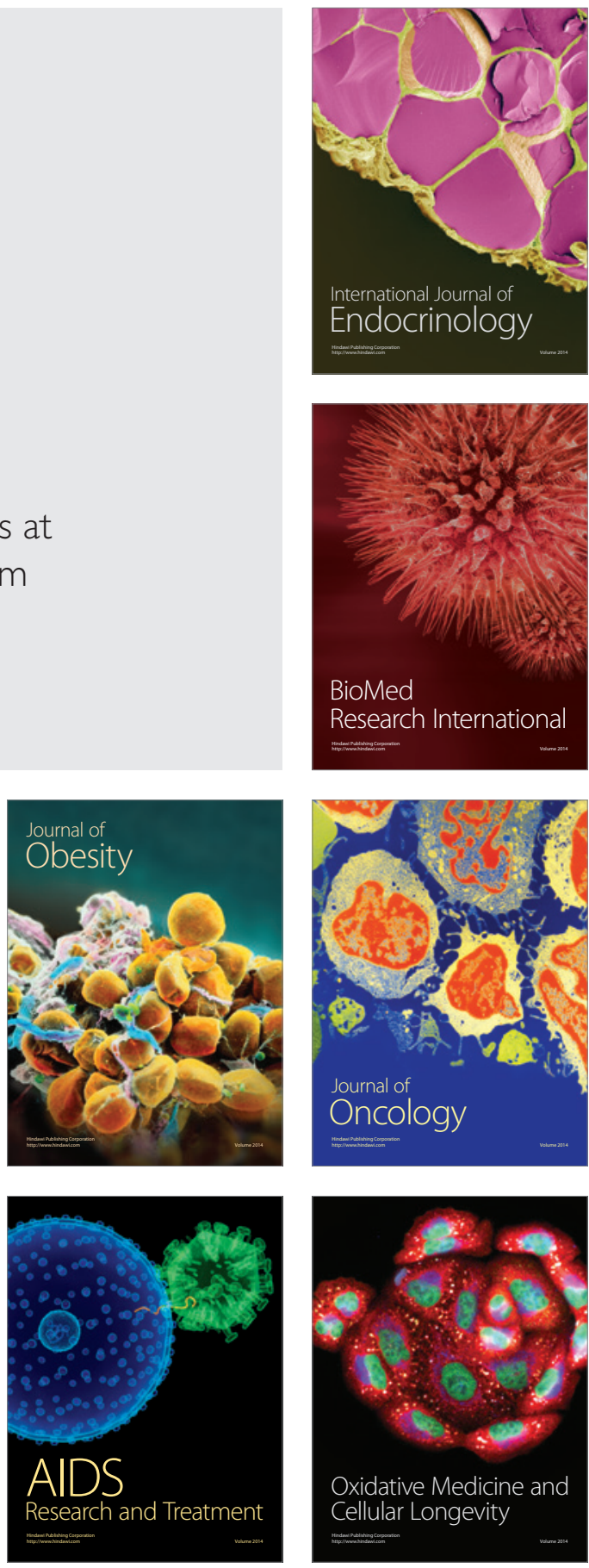